\title{
(2) OPEN ACCESS \\ Percutaneous radiofrequency treatment of the gasserian ganglion for trigeminal neuralgia complicated by trochlear nerve palsy: a case report
}

\author{
Pascal SH Smulders (D) , 1 Michel AMB Terheggen, ${ }^{2}$ José W Geurts (D) , \\ Jan Willem Kallewaard ${ }^{1,2}$
}

'Department of Anesthesiology and Pain Medicine, Amsterdam UMC location AMC, Amsterdam, The Netherlands

${ }^{2}$ Department of Anesthesiology and Pain Medicine, Rijnstate, Arnhem, The Netherlands

Correspondence to Pascal SH Smulders, Department of Anesthesiology and Pain Medicine, Amsterdam UMC location AMC, Amsterdam, The Netherlands;

p.s.h.smulders@amsterdamumc

Received 24 January 2021 Accepted 12 May 2021 Published Online First 26 May 2021
ABSTRACT

Background Trigeminal neuralgia (TN) has the highest incidence of disorders causing facial pain. TN is provoked by benign stimuli, like shaving, leading to severe, short-lasting pain. Patients are initially treated using antiepileptic drugs; however, multiple invasive options are available when conservative treatment proves insufficient. Percutaneous radiofrequency treatment of the trigeminal, or gasserian, ganglion (RF$\mathrm{G}$ ) is a procedure regularly used in refractory patients with comorbidities. RF-G involves complex needle maneuvering to perform selective radiofrequency heat treatment of the affected divisions. We present a unique case of cranial nerve 4 (CN4) paralysis after RF-G.

Case presentation A male patient in his 60 s presented with sharp left-sided facial pain and was diagnosed with TN, attributed to the maxillary and mandibular divisions. MRI showed a vascular loop of the anterior inferior cerebellar artery without interference of the trigeminal complex. The patient opted for RF-G after inadequate conservative therapy. The procedure was performed by an experienced pain physician and guided by live fluoroscopy. The patient was discharged without problems but examined the following day for double vision. Postprocedural MRI showed enhanced signaling between the trigeminal complex and the brainstem. Palsy of CN4 was identified by a neurologist, and spontaneous recovery followed 5 months after the procedure.

Conclusions Mention of postprocedural diplopia in guidelines is brief, and the exact incidence remains unknown. Different mechanisms for cranial nerve (CN) palsy have been postulated: incorrect technique, anatomical variations, and secondary heat injury. We observed postprocedural hemorrhage and hypothesized that bleeding might be a contributing factor in injury of CNs after RF-G.

\section{INTRODUCTION}

Chronic facial pain can be the result of a large number of different conditions. Trigeminal neuralgia (TN) is the disorder with the highest incidence within this group. ${ }^{1} \mathrm{TN}$ is characterized by a severe, short-lasting paroxysmal pain, elicited by stimuli like shaving, cold or eating. The International Headache Society recognizes three distinct subtypes of TN: classical, secondary and idiopathic. Classical TN refers to the subset of patients in whom imaging reveals a neurovascular conflict or vascular compression of the trigeminal nerve, while secondary TN is caused by another illness, like multiple sclerosis. Etiology of idiopathic TN is unidentified. ${ }^{2}$ Although the exact pathophysiology of TN remains unclear, morphological changes to the trigeminal nerve root are observed in a significant proportion of patients. It is speculated that these changes result in focal demyelination, which then leads to unwarranted action potential generation and facial pain. ${ }^{3}$ Incidence is estimated at 12.6 per 100000 per year in the general population and increases with age. It affects men to women in a ratio of 1.0:2.44. ${ }^{1}$

$\mathrm{TN}$ is an often debilitating disease that has a substantial impact on quality of life. ${ }^{4}$ Most patients are treated with antiepileptic drugs, when necessary in combination with other conservative treatments such as psychological therapy. When conservative strategies prove insufficient, a wide palette of interventional procedures are optional as second-line therapy.

Open microvascular decompression (MVD) is associated with the highest rates of morbidity and mortality, and is usually reserved for younger, healthy patients with confirmed neurovascular conflict. Refractory patients with comorbidities can be accepted for less invasive treatment, such as percutaneous radiofrequency treatment of the gasserian ganglion (RF-G) or gamma-knife radiosurgery. Level of evidence for efficacy of these procedures is considered weak to very weak. ${ }^{5}$

RF-G is a technique that allows for selective lesioning of trigeminal nerve divisions. This procedure, first performed by Sweet in 1974, uses realtime fluoroscopy imaging to aid the maneuvering of the needle tip through the foramen ovale to Meckel's cave and the trigeminal ganglion (schematically illustrated in figure 1). ${ }^{6}$ On-site RF-G targets the trigeminal or gasserian ganglion by radiofrequencyinduced heat.

RF-G is generally considered safe and complications are few. In a cohort of 1600 patients, Kanpolat et al reported 225 patients with complications after 2138 RF-G procedures. Diminished corneal reflex was observed in 91 patients (5.7\%), masseter dysfunction in 66 (4.1\%), anesthesia dolorosa and carotid puncture both in 12 patients (each $0.75 \%$ ), keratitis in 10 patients $(0.6 \%)$, cerebrospinal fluid leakage in $2(0.13 \%)$ and meningitis in $1(0.06 \%)$. Transient palsy of a cranial nerve $(\mathrm{CN})$ was present in 12 patients $(0.75 \%)$ (CN3 1 patient, CN6 11 patients). Permanent damage to $\mathrm{CN} 6$ was reported for two patients $(0.13 \%)^{7}$ 


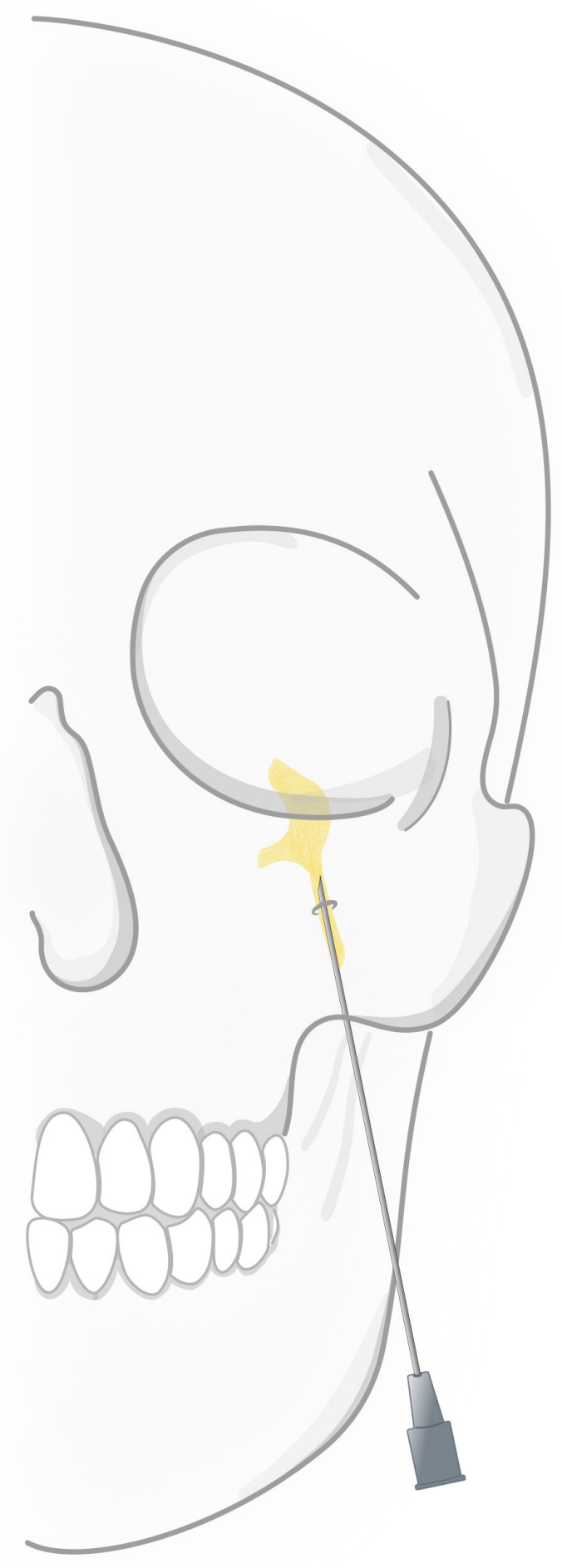

Figure 1 Steep path of the needle tip as it is inserted in the cheek and passes behind the zygomatic bone and through the foramen ovale to the trigeminal ganglion.

$\mathrm{CN}$ palsy is a rare complication of RF-G, and the majority of studies citing $\mathrm{CN}$ palsies date back decades ago. $\mathrm{CN}$ damage after invasive treatment for TN seems not to be linked to one particular $\mathrm{CN}$, and literature is not conclusive to which $\mathrm{CN}$ is most prone to injury. We present a unique case of isolated transient trochlear nerve (cranial nerve 4 (CN4)) paralysis after percutaneous radiofrequency treatment of the gasserian ganglion for TN.

\section{CASE REPORT}

A male patient in his 60 s presented to the outpatient pain clinic with sharp left-sided facial pain. The pain was provoked by washing, chewing, and the application of his continuous positive airway pressure mask. TN was diagnosed in accordance with the International Classification of Headache Disorders. ${ }^{2}$ Symptoms were attributed to the maxillary and mandibular divisions of the trigeminal nerve. Medical history included obstructive sleep apnea, depression, and one incident of probable transient ischemic attack of the brain. Treatment with carbamazepine provided adequate pain relief, but the patient was afraid of consequences to his driving ability with escalating dosage and was hesitant to long-term use.

MRI showed a vascular loop of the anterior inferior cerebellar artery (AICA) without compression or displacement of the left trigeminal complex, and a prominent AICA on the right side. The other (trigeminal) anatomy showed no abnormalities. RF-G of the maxillary and mandibular divisions was scheduled.

The procedure was performed in a specialized outpatient interventional pain center by an experienced pain physician who performs an annual minimum of 20 RF-G procedures. Sedation was administered by a certified nurse sedation practitioner specialist using propofol. Maneuvering of the 22G cannula (SMK Radiofrequency Cannula-straight $10 \mathrm{~cm}$ with $2 \mathrm{~mm}$ tip; St. Jude Medical) was assisted by live fluoroscopy and executed in sterile fashion. Four positions of the cannula were tested under fluoroscopic guidance before acceptable sensory stimulation of the maxillary and mandibular divisions was attained. The final, and most cephalad, position of the needle tip was slightly past the angle formed by the petrosal ridge of the temporal bone and the clivus. Stimulation was performed awake to confirm correct needle positioning. As the ultimate sensory threshold of $0.3 \mathrm{~V}$ was on the edge of the target range, radiofrequency lesioning was performed directly at $70^{\circ} \mathrm{C}$ during $60 \mathrm{~s}$, instead of starting at $60^{\circ} \mathrm{C}$. Motor response was absent at $2.0 \mathrm{~V}$. The sensory threshold rose from 0.3 to $0.8 \mathrm{~V}$ after the lesion was applied, and no subsequent lesions were made. Postoperative corneal reflex was intact; vision and facial sensibility were normal.

The day following the procedure, the patient complained of double vision and was examined at the emergency department by a neurologist. Diplopia was most pronounced when looking down. Monocular eyesight was normal, facial pain was absent. Postprocedural MRI of the brain showed enhanced signaling on the procedural side between the trigeminal ganglion and the brainstem (figure 2), and (asymptomatic) sinusitis of the maxillary and sphenoid sinus. Ophthalmic consultation established a restriction in function of the left superior oblique muscle, which is innervated by CN4. Prism correction proved unhelpful and otolaryngology examination gave no new insights. Trochlear nerve function spontaneously recovered to normal 5 months after the procedure.

The patient approved publication of the case history.

\section{DISCUSSION}

Large, well-established trials on outcome after treatment for TN are few. As such, most data on complication risk are derived from a select number of decades old cohort studies. The incidence of $\mathrm{CN}$ paralysis after RF-G is reported to be between $0.29 \%$ and 


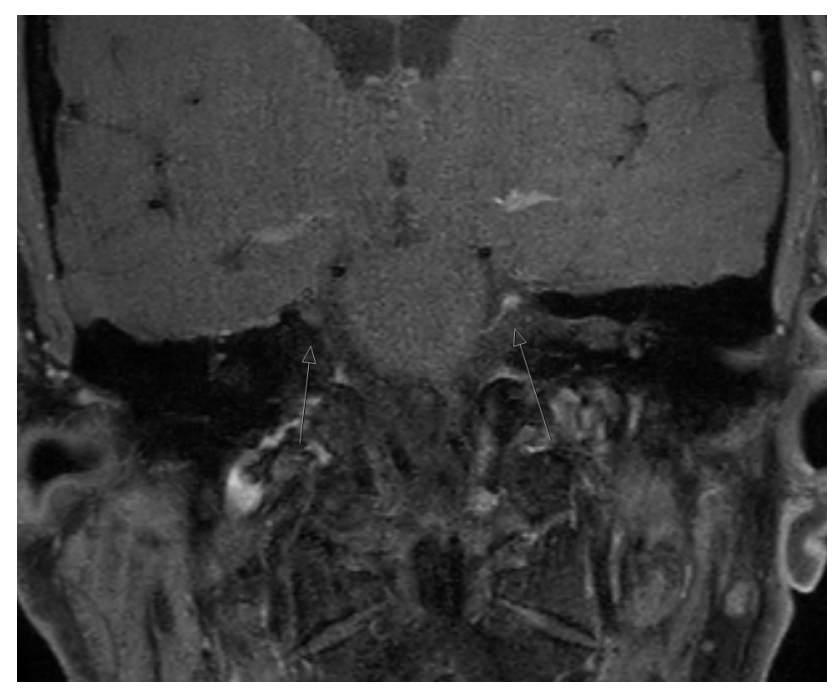

Figure 2 Postprocedural MRI of the brain showing enhanced signaling on the procedural side. The arrows point to the location of enhanced signalling (ie the hematoma) of the MRI.

$7.7 \%$, with more recent studies citing numbers at the lower end of this range. ${ }^{7-12}$ These studies are regularly referenced in guidelines, but mention of postprocedural diplopia is mostly lacking. Although the incidence of diplopia after RF-G remains unclear, clinical experience confirms that occurrence of $\mathrm{CN}$ palsy after RF-G is exceedingly rare.

A handful of case reports concerning $\mathrm{CN}$ palsy after treatment for TN have been published over the last decennia. Treatment modalities for these patients span all procedures, except for gamma-knife surgery. Multiple authors report isolated transient CN4 or CN6 palsy after (repeated) percutaneous balloon compression, RF-G, or MVD. ${ }^{13-18}$ One study documents a patient with permanent trochlear nerve damage and corresponding diplopia after RF-G. ${ }^{19}$ Subarachnoid hemorrhage after attempted RF-G, followed by - among other symptoms multiple bilateral CN paralysis, has also been reported. ${ }^{20}$

Preprocedural imaging has become standard practice, ensuring clinicians of knowledge of a patient's anatomy. Preprocedural MRI has an important role in finding the cause of TN and may detect anatomical variations important to consider when planning invasive procedures. However, a certain level of imprecision or ambiguity is inherent to imaging studies as the trigeminal complex and Meckel's cave are relatively small anatomical structures and can usually only be partially visualized. The postprocedural diagnosis of an arteriovenous malformation in a patient with CN4 palsy after percutaneous balloon compression demonstrates this imprecision and illustrates that preprocedural MRI is unable to prevent all cases of CN palsy. ${ }^{21}$

The trochlear nerve has the longest intracranial course of all $\mathrm{CNs}$ and is the only one to originate from the dorsal midbrain. It decussates in the superior medullary velum, transverses the brain and enters the cavernous sinus to terminate on the superior oblique muscle. ${ }^{22}{ }^{23}$ The decussating path of the trochlear nerve allowed the conclusion that the trochlear nerve, not the trochlear nucleus, was injured in this case history. The cavernous sinus is a dural envelope that contains not only $\mathrm{CN} 4$, but also CN3 and CN6. ${ }^{23}$ Its boneless makeup and close relation to Meckel's cave may play a role in the mechanism underlying $\mathrm{CN}$ paralysis after RF-G.

Various mechanisms for $\mathrm{CN}$ injury have been postulated, of which the most prominent is incorrect procedural technique.
The proximity of the trigeminal complex to the different $\mathrm{CNs}$ warrants extreme caution, as minute displacement of the cannula in either depth or direction may lead to complications. The application of fluoroscopy enables real-time needle visualization and is thought to be crucial to minimize the incidence of adverse events.

Different authors hypothesize that the origin of $\mathrm{CN}$ palsy is equipment-related, that is, insertion too far into Meckel's cave, pertaining to cannula design, or overinflation in the setting of balloon compression. ${ }^{1617192425}$ Other mentioned causes of CN damage are anatomical variations and secondary heat injury originating from the outward radiation of thermal energy from the target site. ${ }^{16181921}$

We propose a novel explanation and hypothesize that procedure-related hemorrhage might lead to compression and injury of CNs. We found enhanced postprocedural magnetic resonance signaling in this patient and presume this to be a reflection of hematoma. Hemorrhage might be the causative mechanism for CN4 palsy in this case, but other explanations could be (undetected) anatomical variations or secondary heat injury.

In conclusion, trochlear nerve palsy resulting in diplopia is a rare, generally transient complication of percutaneous radiofrequency treatment of the gasserian ganglion. Preprocedural imaging is essential for finding the origin of $\mathrm{TN}$ and contributes to risk assessment when planning invasive treatment techniques, while awake stimulation and careful needle maneuvering under fluoroscopic guidance are needed for correct application of RF-G.

Acknowledgements We especially thank Simone Kersten for her graphical illustration demonstrating the cannula path toward the trigeminal ganglion (figure 1).

Contributors All authors contributed to writing the article.

Funding The authors have not declared a specific grant for this research from any funding agency in the public, commercial or not-for-profit sectors.

Competing interests None declared.

Patient consent for publication Not required.

Provenance and peer review Not commissioned; externally peer reviewed.

Open access This is an open access article distributed in accordance with the Creative Commons Attribution 4.0 Unported (CC BY 4.0) license, which permits others to copy, redistribute, remix, transform and build upon this work for any purpose, provided the original work is properly cited, a link to the licence is given, and indication of whether changes were made. See: https://creativecommons.org/ licenses/by/4.0/.

\section{ORCID iDs}

Pascal SH Smulders http://orcid.org/0000-0001-8433-2050

José W Geurts http://orcid.org/0000-0002-7043-968X

\section{REFERENCES}

1 Koopman JSHA, Dieleman JP, Huygen FJ, et al. Incidence of facial pain in the general population. Pain 2009;147:122-7.

2 Headache Classification Committee of the International Headache Society (IHS). The International classification of headache disorders. 3 edn. Cephalalgia, 2018: 1-211.

3 Maarbjerg S, Di Stefano G, Bendtsen L, et al. Trigeminal neuralgia - diagnosis and treatment. Cephalalgia 2017:37:648-57.

4 Tölle T, Dukes E, Sadosky A. Patient burden of trigeminal neuralgia: results from a cross-sectional survey of health state impairment and treatment patterns in six European countries. Pain Pract 2006;6:153-60

5 Huygen F, Kallewaard JW, van Tulder M, et al. "Evidence-based interventional pain medicine according to clinical diagnoses": update 2018. Pain Pract 2019;19:664-75.

6 Sweet WH, Wepsic JG. Controlled thermocoagulation of trigeminal ganglion and rootlets for differential destruction of pain fibers. 1. trigeminal neuralgia. J Neurosurg 1974;40:143-56

7 Kanpolat Y, Savas A, Bekar A, et al. Percutaneous controlled radiofrequency trigeminal rhizotomy for the treatment of idiopathic trigeminal neuralgia: 25-year experience with 1,600 patients. Neurosurgery 2001;48:524-34. 
8 Mittal B, Thomas DG. Controlled thermocoagulation in trigeminal neuralgia. J Neurosurg 1986;49:932-6.

9 Onofrio BM. Radiofrequency percutaneous gasserian ganglion lesions. results in 140 patients with trigeminal pain. J Neurosurg 1975;42:132-9.

10 Tew JM, Keller JT. The treatment of trigeminal neuralgia by percutaneous radiofrequency technique. Clin Neurosurg 1977:24:557-78.

11 van Loveren H, Tew JM, Keller JT, et al. A 10-year experience in the treatment of trigeminal neuralgia. Comparison of percutaneous stereotaxic rhizotomy and posterior fossa exploration. J Neurosurg 1982;57:757-64.

12 Broggi G, Franzini A, Lasio G, et al. Long-term results of percutaneous retrogasserian thermorhizotomy for "essential" trigeminal neuralgia: considerations in 1000 consecutive patients. Neurosurgery 1990;26:783-7.

13 Chatterjee N, Chatterjee S, Roy C. Abducens nerve palsy after percutaneous radiofrequency ablation of gasserian ganglion. J Neurosurg Anesthesiol 2014;26:89-90.

14 Choudhari KA. Isolated abducent nerve palsy after microvascular decompression for trigeminal neuralgia: case report. Neurosurgery 2005:57:E1317.

15 Marchi C, de Aguiar PHP, Moura AM, et al. Abducent nerve palsy after microballoon compression of the trigeminal ganglion: case report. Surg Neurol Int 2017;8:125.

16 Urculo E, Alfaro R, Arrazola M, et al. Trochlear nerve palsy after repeated percutaneous balloon compression for recurrent trigeminal neuralgia: case report and pathogenic considerations. Neurosurgery 2004;54:505-9.
17 Harrigan MR, Chandler WF. Abducens nerve palsy after radiofrequency rhizolysis for trigeminal neuralgia: case report. Neurosurgery 1998;43:623-5.

18 Grimson BS, Boone SC. Sixth nerve palsy complicating percutaneous thermal ablation of the trigeminal nerve rootlet. Am J Ophthalmol 1981;92:225-9.

19 Yemisci M, Dogulu CF, Kansu T, et al. Trochlear nerve palsy as a complication of trigeminal radiofrequency rhyzolysis. Neuroophthalmology 2000;24:485-7.

20 Madhusudan Reddy KR, Arivazhagan A, Chandramouli BA, et al. Multiple cranial nerve palsies following radiofrequency ablation for trigeminal neuralgia. $\mathrm{Br} J$ Neurosurg 2008;22:781-3.

21 Lichtor T, Mullan JF. A 10-year follow-up review of percutaneous microcompression of the trigeminal ganglion. J Neurosurg 1990;72:49-54.

22 Joo W, Rhoton AL. Microsurgical anatomy of the trochlear nerve. Clin Anat 2015;28:857-64

23 Yasuda A, Campero A, Martins C, et al. Microsurgical anatomy and approaches to the cavernous sinus. Neurosurgery 2008;62:0NS-4-ONS-27.

24 Brown JA, Chittum CJ, Sabol D, et al. Percutaneous balloon compression of the trigeminal nerve for treatment of trigeminal neuralgia. Neurosurg Focus 1996; : E10

25 Tobler WD, Tew JM, Cosman E, et al. Improved outcome in the treatment of trigeminal neuralgia by percutaneous stereotactic rhizotomy with a new, curved tip electrode. Neurosurgery 1983;12:313-7. 